\title{
Differential effects of ventral and regional dorsal striatal lesions on sucrose drinking and positive and negative contrast in rats
}

\author{
DAWN M. EAGLE, TREVOR HUMBY, MARY HOWMAN, \\ ANNA REID-HENRY, STEPHEN B. DUNNETT, and TREVOR W. ROBBINS \\ University of Cambridge, Cambridge, England
}

\begin{abstract}
The striatum mediates several different components of appetitive ingestive behavior in a regiondependent manner. Previous work has implicated the dorsal and ventral striatum in both consummatory and appetitive aspects of motivated behavior. In this study, we compared the effects of excitotoxic, fiber-sparing lesions of the ventral, medial, and lateral striatum on consummatory responses to sucrose solution rewards. Consummatory responses were measured in terms of volume consumption and licking behavior, and in a successive contrast paradigm. Ventral striatal lesions shifted the intake-concentration function to the left but did not affect positive or negative contrast. Lateral striatal lesions reduced volume consumption, apparently as a result of motoric impairments of licking efficiency, but had no significant motivational effects. The effects of medial striatal lesions were less clear cut, but they probably included both motor and subtle motivational deficits.
\end{abstract}

Considerable evidence supports a role for the ventral striatum in appetitive behavior (Robbins \& Everitt, 1996). Such evidence comes from studies of striatal involvement in the reinforcing effects of intracranial stimulation and abused drugs, such as the psychomotor stimulants (Koob, 1992; Koob \& Nestler, 1997; Robbins \& Everitt, 1996; Wise \& Bozarth, 1987), and also from studies of appetitive behavior directed toward natural rewards such as food, sex, and novelty (Burns, Annett, Kelley, Everitt, \& Robbins, 1996; Everitt, 1990; Mitchell, 1994; Robbins, Cador, Taylor, \& Everitt, 1989; Robbins \& Everitt, 1992). Within the striatum, distinct dorso-ventral regional differentiation of motivational function is likely, since the dorsal striatum is proposed to be involved in the sensorimotor coordination of consummatory behavior (Berridge \& Cromwell, 1990; Brown \& Robbins, 1989; Dunnett \& Iversen, 1980; Ungerstedt, 1971) and the ventral striatum is considered important in determining the control of appetitive aspects of behavior (Robbins \& Everitt, 1992).

The exact role of the striatum in motivational processes is complicated, however, by the preponderance of studies of its dopaminergic innervation (Dunnett \& Robbins, 1992). Dopamine depletion from the dorsal striatum results in deficits in the capacity to initiate and execute forms of consummatory behavior (Bakshi \& Kelley, 1991a, 1991b). For example, 6-OHDA lesions of the substantia nigra/VTA produce profound aphagia and adipsia, effects

This research was supported by a Medical Research Council Programme Grant awarded to S.B.D., T.W.R., and B.J.E. Correspondence concerning this article should be sent to D. Eagle, MRC Cambridge Centre for Brain Repair, University of Cambridge, Forvie Site, Robinson Way, Cambridge CB2 2PY, England (e-mail: de102@hermes. cam.ac.uk). which provide evidence that dopamine depletion in this region induces a disturbance of the sensorimotor integration of ingestive behavior and impairments of the motivational properties of primary rewards, such as food and water (Marshall, Richardson, \& Teitelbaum, 1974; Zigmond \& Stricker, 1972).

Berridge and Robinson (1998) and Salamone, Cousins, and Snyder (1997) have reviewed the involvement of dopaminergic mechanisms in reward-related behaviors in detail. Evidence suggests that both appetitive and consummatory components of behavior involve the nucleus accumbens and that the components are separable by manipulation of dopamine and opiates within this region of the brain (Kelley, Bless, \& Swanson, 1996; Zhang \& Kelley, 1997). Dopamine depletion from the ventral striatum also leads to a reduction in spontaneous motor activity, which is a finding that could indicate a reduction in the appetitive response to reward (Bakshi \& Kelley, 1991a; Cousins \& Salamone, 1994; Cousins, Sokolowski, \& Salamone, 1993; Koob, Riley, Smith, \& Robbins, 1978). Moreover, dopamine is considered important for eating, drinking, and orofacial motor control (Kelley, Gauthier, \& Lang, 1989).

The effects of manipulations of the mesolimbic dopamine system on sucrose consumption are complex, and consequently, it is difficult to support a simple hypothesis of the role of the mesolimbic dopamine system in sucrosedrinking behavior. In some studies, for example, infusions of dopamine receptor antagonists into the ventral striatum have produced a rightward shift in sucrose consumption (Phillips, Willner, \& Muscat, 1991a, 1991b, 1991c), but, in other studies, the infusions have had no effect on the intake of higher concentrations of sucrose (Ikemoto \& Panksepp, 1996; Muscat \& Willner, 1989). 
The precise roles of the dorsal and ventral striatum in incentive motivation, distinct from their dopaminergic innervation, are unclear. Balleine and Killcross (1994) found that when they manipulated the incentive value of the outcome of an operant leverpressing task, rats with ventral striatal excitotoxic lesions were sensitive to these changes on certain aspects of the task, but not on others. Within the dorsal striatum, regional functional differentiation may also exist, which could relate to differences in appetitive and consummatory responses. For example, considerable experimental evidence locates neurones within the lateral dorsal striatum that are specific to tongue and mouth movement (Mittler, Cho, Peoples, \& West, 1994) and potentially to the control of responses to food. The role of the medial dorsal striatum is less clear, but studies indicate a continuum of function from the ventral striatum, through the medial striatum and into the lateral striatum (Whishaw, Mittleman, Bunch, \& Dunnett, 1987). In addition, considerable connectivity exists between both the medial and lateral striatum and other brain regions that are more clearly implicated in reward mechanisms (Alexander \& Crutcher, 1990; Alheid \& Heimer, 1998).

In this study, we examined the ingestive responses of rats with different striatal lesions to variations in sucrose concentration. Concentration-dependent shifts to the left or to the right that were produced by the lesions suggest motivationally dependent deficits; both parallel shifts and unchanged asymptotes were evidence against simple motor effects. We also assessed the dynamic response to shifts in anticipated sucrose concentrations with a successive contrast procedure that measured both positive and negative contrast.

Considerable evidence supports a motivationalemotional interpretation of contrast, or shifts in reward magnitude (Flaherty, 1982). Problems arise with any attempt to equate the behavioral properties of incentive contrast with emotion, but analysis of this behavior can provide important information about the contribution of different regions of the brain to reward evaluation (Robbins \& Everitt, 1992). Actual evidence of the effects on sucrose-related contrast is limited to a few studies. Lesions of the parabrachial nuclei (Grigson, Spector, \& Norgren, 1994) and amygdala (Becker, Jarvis, Wagner, \& Flaherty, 1984; Salinas, Packard, \& McGaugh, 1993) are effective in eliminating successive negative contrast effects, but there are no effects of disruption to hippocampal activity (Flaherty, Rowan, Emerich, \& Walsh, 1989 ) or to serotonergic mechanisms (Flaherty et al., 1990) on negative contrast. Both of the latter structures have specific projections to the striatum, but the effects of any disruption to the striatum itself on these forms of motivated behavior are not known.

Therefore, in this study we evaluated the effects of excitotoxic lesions to the striatum on consummatory behavior over a range of reinforcer magnitudes, incorporated the effects of food deprivation on this behavior, and investigated positive and negative contrast effects. This has enabled us to compare effectively the roles of the dorsal and ventral regions of the striatum in appetitive behavior.

\section{METHOD}

\section{Subjects}

The subjects were 32 male hooded Lister rats (Harlan; Bicester, England) that weighed $315-360 \mathrm{~g}$ at the start of the study. The rats were housed in groups of 4 under a reversed 12:12-h light:dark cycle (lights off at $0930 \mathrm{~h}$ ) and were tested between 1100 and $1800 \mathrm{~h}$ during the dark phase of this cycle. Body weights were maintained at approximately $95 \%$ of the free-feeding weight by feeding with 15-20 g of expanded laboratory chow $1 \mathrm{~h}$ after the end of each daily test session. Water was available ad lib, except during the experimental session.

\section{Surgery}

The rats were randomly allocated into four groups. They received bilateral medial striatal lesions $(n=8)$, lateral striatal lesions $(n=$ $8)$, ventral striatal lesions $(n=8)$, or sham surgery $(n=8)$ divided among medial (3), lateral ( 3 ), and ventral (2) sites. The rats were deeply anesthetized (with $1.5 \%$ halothane in $2: 1 \mathrm{O}_{2}$ and $\mathrm{N}_{2} \mathrm{O}$ ), and bilateral lesions were made by infusion of $0.09 \mathrm{M}$ quinolinic acid (Sigma) in phosphate-buffered saline (PBS), $\mathrm{pH}=7.4$, through a 30-gauge stainless steel cannula connected with polyethylene tubing to a $10-\mu \mathrm{l}$ glass syringe in a microdrive pump. For medial and lateral striatal lesions, $2 \times 0.35-\mu$ infusions were made on each side, and at each lesion site, the infusion was made at two depths ventrally. For ventral lesions, one single-depth $0.7-\mu l$ infusion was made on each side. Quinolinic acid was infused at a rate of 0.125 $\mu \mathrm{l} / \mathrm{min}$. (For medial and lateral lesions, it was infused for $1 \mathrm{~min}$ $20 \mathrm{sec}$ at the more ventral coordinate; then the cannula was raised, and infusion was continued for the remainder of the infusion time, to $2 \mathrm{~min} 48 \mathrm{sec}$.) Lesion coordinates for the medial striatum were anterior $(A)=0.2 \mathrm{~mm}$ to bregma, lateral $(\mathrm{L})= \pm 2.0 \mathrm{~mm}$ to the midline, vertical $(\mathrm{V})=5.0 \mathrm{~mm}$ and $4.0 \mathrm{~mm}$ below dura, and $\mathrm{A}=1.2$, $\mathrm{L}= \pm 2.0, \mathrm{~V}=5.0,4.0$. For the lateral striatum, lesion coordinates were $\mathrm{A}=-0.3, \mathrm{~L}= \pm 3.6, \mathrm{~V}=5.5,5.0$ and $\mathrm{A}=0.7, \mathrm{~L}= \pm 3.6, \mathrm{~V}=$ $5.0,4.5$. For the ventral striatum, lesion coordinates were $A=1.6$, $\mathrm{L}= \pm 1.6, \mathrm{~V}=6.8$. The incisor bar was set $2.3 \mathrm{~mm}$ below the interaural line. Following a further $1 \mathrm{~min}$ for diffusion, the cannula was retracted and the wound was cleaned and sutured. The control animals received identical injections of $\mathrm{PBS}, \mathrm{pH}=7.4$. Postoperatively, all rats were given $1.25 \mathrm{mg} / 0.25 \mathrm{ml}$ i.p. diazepam (CP Pharmaceuticals, England) and paracetamol (Unichem; $250 \mathrm{mg}$ dissolved in $500 \mathrm{ml}$ water). One of the ventrally lesioned group died following surgery, an event that reduced the group size to 7 .

\section{Apparatus}

Sessions were performed in four drinking chambers $(550 \times 380$ $\times 450 \mathrm{~mm}$ ) with black Perspex walls, clear Perspex lid, and shockgrid stainless steel flooring. Sucrose solutions were presented in 50 $\mathrm{ml}$ burettes attached to stainless steel ball-tipped drinking spouts. These spouts were presented through holes in one wall of the chamber; the tip of the spout was $25 \mathrm{~mm}$ above the floor and $30 \mathrm{~mm}$ from the wall. The spouts were encased in plastic; the tip of the spout was retracted $4 \mathrm{~mm}$ inside the plastic sleeve. This prevented the rats from making continuous contact with the metal spout and enabled individual licks to be measured. When a rat stood on the stainless steel flooring and made contact with the licking tube, a circuit was completed, which generated a signal that was recorded on an Acorn A5000 microcomputer. Volumes consumed per trial were read from 
the burette to an accuracy of $0.05 \mathrm{ml}$. Access to the drinking spouts was controlled by raising or lowering a Perspex panel $50 \mathrm{~mm}$ in front of the spouts or by withdrawing the drinking tube from the box.

\section{Experimental Procedure}

The rats were presented with $0.2 \mathrm{M}$ sucrose in their home cages for $2 \mathrm{~h}$ on each of 2 days during the 14-day postsurgery recovery period. Following this, the rats were habituated to the drinking boxes in daily sessions with $2 \mathrm{~min}$ in the box without access to sucrose, followed by a 20 -min drinking session, during which $0.2 \mathrm{M}$ sucrose was available at both of two tubes that were presented. Habituation continued for 7 days, when the volumes of consumed sucrose had reached a stable level.

Experiment 1: Sucrose consumption of food-deprived rats. The rats received two-tube presentations: One tube contained test solution, and the other contained water. Each solution strength was presented for two days, with at least $24 \mathrm{~h}$ between the presentation of solutions of different strengths. Six test solutions were presented $(0.0,0.05,0.1,0.2,0.4$, and $0.8 \mathrm{M}$ sucrose). To control for any possible position effects, the tubes were assigned randomly to their presentation positions. To control for any possible effects of positive or negative contrast between concentrations, the solutions were presented to half the rats on an ascending-descending (AD) scale of concentrations and to the other half on a descending-ascending (DA) scale. (Each rat received each two-tube pairing for 2 days ascending and 2 days descending.) Rats were tested 4 days per week ( 2 days on, 1 day off, 2 days on, 2 days off), and testing was complete within 44 days of the first trial. Each rat's weight was recorded for each test session.

Experiment 2: Sucrose consumption and the effects of prefeeding. In order to assess the effects of hunger levels on performance, the rats were returned to ad-lib feeding for $1 \mathrm{~h}$ before experimental trials and then tested on the ascending regime of sucrose presentation.

Experiment 3: Affective contrast paradigm. We examined the effects of striatal lesions on the response to within-session successive negative and positive contrast. Single-tube presentations were made in a $30-\mathrm{min}$ trial, during which the tube was available for six 1 -min drinking periods. The drinking periods were separated by 4 min rest periods, during which the tube was withdrawn from the box. We started timing the 1 -min drinking period from the first lick at the tube. The rats received one test solution for Drink Periods 1-3 and a different solution for Drink Periods 4-6. For negative contrast trials, $0.2 \mathrm{M}$ sucrose was switched to $0.05 \mathrm{M}$ sucrose; for positive contrast trials, $0.0125 \mathrm{M}$ sucrose was switched to $0.05 \mathrm{M}$ sucrose; and for the no contrast trials, $0.05 \mathrm{M}$ sucrose was presented for each of the six drink periods, but the tube was switched after the third drink period to control for tube-changing effects in the other two trials. Each rat received one positive contrast, one negative contrast, and one no contrast session. Testing took place over 5 days on alternate days. The order of presentation of positive, negative, and no contrast was randomized. Each rat's weight was recorded for each test session.

\section{Statistical Analyses}

For Experiments 1 and 2, the data were analyzed with repeated measures ANOVA, using the Genstat 5 statistical package (NAG, Oxford, England). Concentration was a within-subjects factor, and lesion group was a between-subjects factor. A preliminary analysis of data under food-deprived conditions also included schedule (AD or DA) and order (A or D) as between-subjects factors, but this analysis gave rise to no significant differences, so for simplification of the second part of the study, the data are presented only for the ascending order of presentation. Post hoc analyses were performed by using Dunnett's $t$ test for comparisons between the lesion groups and the sham lesioned controls and by using Šidák's test for multi- ple independent pairwise comparisons between conditions within groups. For cross-experiment analysis of the effect of prefeeding, an additional ANOVA was carried out to include food as a withinsubjects factor.

For Experiment 3, the data were analyzed for the first postshift drinking period (Period 4). Lesion was a between-subjects factor, and contrast was a within-subjects factor.

\section{Histological Analysis}

After behavioral testing was completed, the rats were deeply anesthetized by intraperitoneal injection of $0.7 \mathrm{ml}$ of sodium pentobarbitone (Euthatal; May \& Baker, Harlow, England) and were transcardially perfused with approximately $100 \mathrm{ml} \mathrm{PBS,} \mathrm{pH}=7.4$, followed by $250 \mathrm{ml} \mathrm{4 \%}$ paraformaldehyde in PBS. The brains were removed and post-fixed in 4\% paraformaldehyde for $24 \mathrm{~h}$ and then transferred to $25 \%$ sucrose in PBS until they sank. The tissue was serially sectioned at $60 \mu \mathrm{m}$ on a freezing-stage sledge microtome, and two parallel 1:6 series were mounted on slides. One series was stained with cresyl violet, and the other for acetylcholinesterase (AChE) activity. Histological sections were visualized microscopically under conventional bright field illumination and photographed digitally, and photomicrographs were prepared with the use of Adobe Photoshop 3.0.

\section{RESULTS}

\section{Postoperative Recovery}

The animals were allowed to recover for 14 days after being lesioned, and then food intake was restricted for 7 days before testing began. Several animals with ventral lesions were hypophagic and suffered significant postsurgical weight loss. Consequently, the mean weight of the ventral lesion group was consistently lower than that of the control group throughout the experiment. The presurgery mean weights (g) were as follows: control, 336.3; medial, 336.3; lateral, 343.8; and ventral, 336.9. The postsurgery mean weights $\pm S E M(\mathrm{~g})$ were as follows: control, $347.7 \pm 2.5 ;$ medial, $346.8 \pm 2.5$; lateral, 350.3 \pm 2.9 ; and ventral, $333.5 \pm 2.0$. However, when weight was used as a covariate in the analyses, the significance of the outcome was not affected for any measure, and so for clarity, the unadjusted data are presented.

\section{Histology}

Figure 1 shows the extent of minimum and maximum striatal damage for the medial, lateral, and ventral lesion groups. In all cases, there was considerable cell death in the lesioned regions, accompanied by striatal shrinkage, effects which can be seen in the sections shown in Figure 2 . The ventral lesions were more variable in position and extent of damage than were the medial or lateral striatal lesions. In all ventral lesions there was some damage to both the shell and core of the nucleus accumbens, although in some cases the damage also extended to the diagonal band and the ventral pallidum.

\section{Experiment 1:}

\section{Sucrose Consumption of Food-Deprived Rats}

Lesions of the ventral, lateral, and medial striatum all had significant effects on sucrose intake, with dissociation 

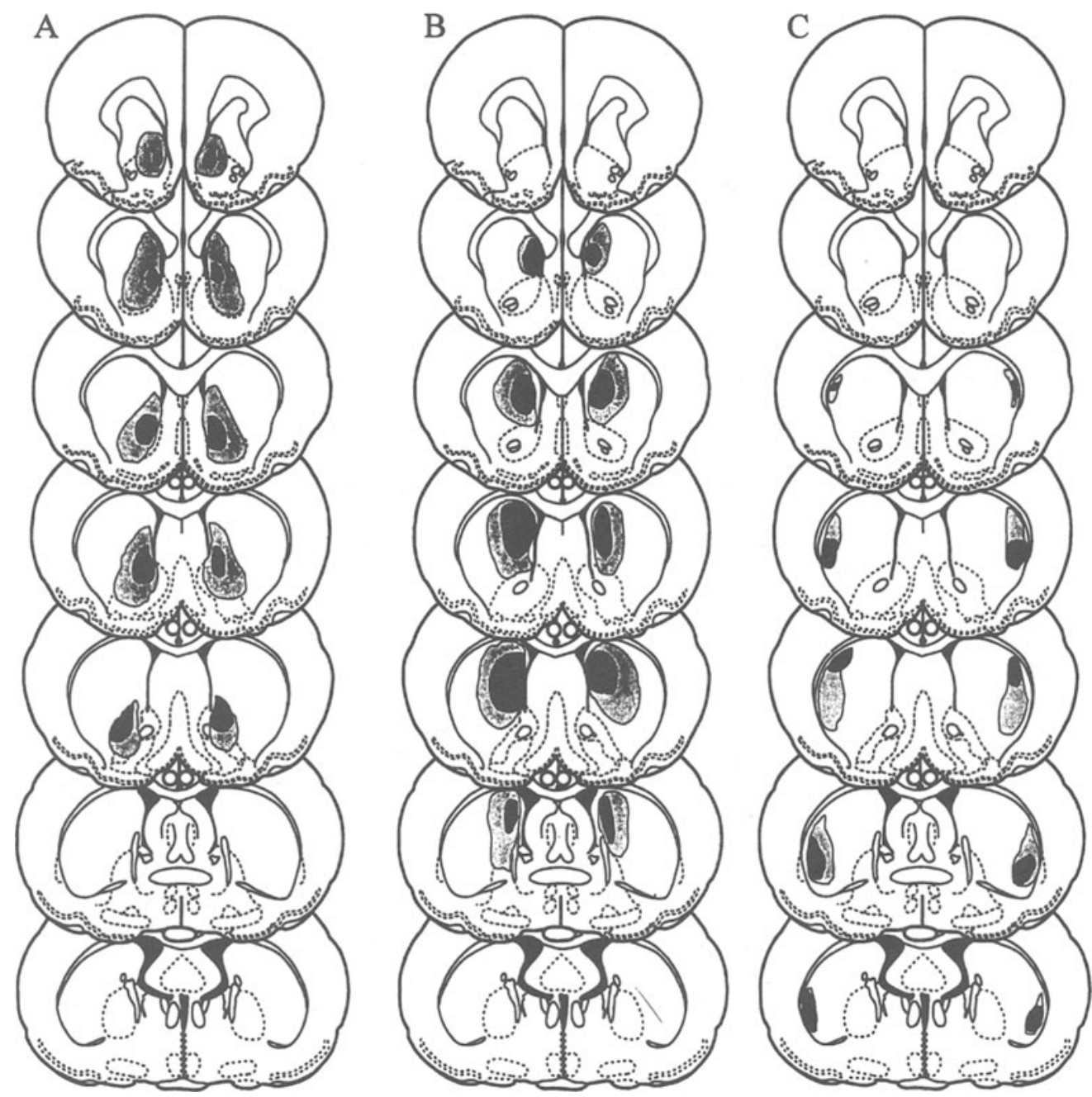

Figure 1. Schematic representation of bilateral quinolinic acid lesions of (A) the ventral striatum, (B) the medial striatum, and $(C)$ the lateral striatum. The black areas represent the smallest, and the stippled areas, the largest, regions of neuronal loss observed in the lesion group.

between lesion groups in the pattern of effect on sucrose drinking. As shown in Figure 3A, ventral striatal lesions produced a significant leftward shift in the inverted Ushaped intake-concentration curve, increasing the intake of weak sucrose solutions $(0.1 \mathrm{M})$ and decreasing the intake of the stronger solutions $(0.4 \mathrm{M}$ and $0.8 \mathrm{M})$ [lesion $\times$ concentration $F(15,130)=4.47, p<.001]$. The peak intake was a concentration of $0.1 \mathrm{M}$ by the animals with ventral lesions, compared with $0.2-0.4 \mathrm{M}$ by the control animals and the other lesion groups. The overall intake volume by the animals with ventral striatal lesions was not affected, compared with the overall intake volume by the control animals. In contrast, medial and lateral striatal lesions both significantly decreased the volume intake of sucrose by the rats [lesion $F(3,26)=3.30, p<$ .05 ; Dunnett's $t$ test $p<.05$ for the medial and lateral groups], without shifting the shape of the intake func- tion, which remained the same as for the control animals. Figure $3 \mathrm{~A}$ shows that the decrease in volume intake was greater for laterally lesioned rats than it was for medially lesioned rats, and that for both lesion groups, but especially for the medially lesioned group, the decrease in volume intake was greater at higher sucrose concentrations.

Licking behavior. Analysis of licking behavior also clearly indicated a dissociation between lesion groups, with lateral lesions producing a great increase in licking activity [see Figure 3B; lesion $F(3,26)=6.04, p<.01$ ] . This effect was greatest at the concentrations around peak intake [lesion $\times$ concentration $F(15,130)=2.57, p<$ .01 ; Dunnett's $t$ test $p<.05$ for the lateral-control comparison at $0.1 \mathrm{M}, 0.2 \mathrm{M}$, and $0.4 \mathrm{M}$ ]. Medial striatal lesions increased licking activity at only the concentration of peak intake (Dunnett's $t$ test $p<.05$ for medial control comparison at $0.2 \mathrm{M}$ only). The licking behavior 

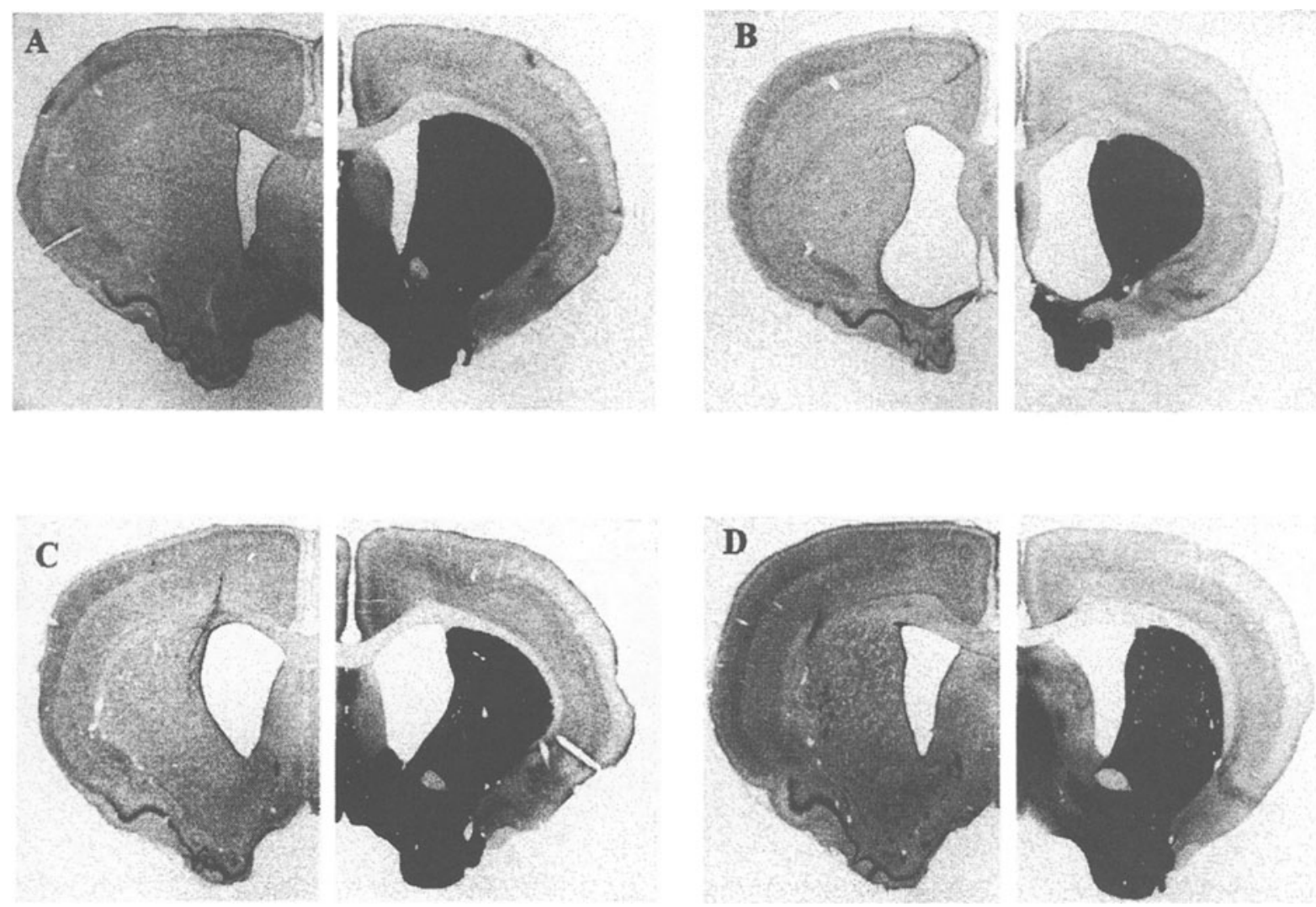

Figure 2. Photomicrographs of coronal sections of rat brains with (A) sham lesion, lesions to (B) the ventral striatum, (C) the medial striatum, and (D) the lateral striatum. Each section shows two sections from the same animal, stained with cresyl violet (left side) and for acetylcholinesterase activity (right side).

of the rats was affected very little by ventral striatal lesions, compared with the licking behavior of the control animals.

Analysis of the derived measure of lick volume (total volume/total number of licks; see Figure 3C) showed that the animals with lateral striatal lesions had significantly reduced lick volumes, compared with the lick volumes of the control animals [lesion $F(3,26)=8.42, p<$ $.001]$. The ventrally lesioned animals showed no differences from the control animals on this measure, and in both groups, lick volume appeared to vary with total volume intake. The medially lesioned animals were somewhere in between the other two lesion groups, with a small, but overall nonsignificant, reduction in lick volume.

Preference for sucrose over water. None of the lesions had any significant effect on the rats' preference for sucrose over water in trials in which sucrose and water were presented simultaneously [see Figure 3D; lesion $F(3,26)=1.90$, n.s.], and this was the case for all the concentrations tested [lesion $\times$ concentration $F(15,130)=$ 1.17, n.s.]. Across all concentrations, the mean preference for sucrose over water was high, over $97 \%$, with water consumption during the 20 -min trials rarely more than $0.2 \mathrm{ml}$.

\section{Experiment 2: Sucrose Consumption and the Effects of Prefeeding}

For all groups, the effect of pretrial feeding was the same: a considerable reduction in intake, compared with that seen in the food-deprived tests [see Figure 4A; food $F(1,26)=222.13, p<.001]$, and a flattening of the intake-concentration curve. However, the ventral lesion effect of shifting the intake-concentration curve to the left was still evident, with significantly greater intake at 0.1 and $0.2 \mathrm{M}$ [lesion $\times$ concentration $F(3,150)=1.78, p<$ $.05]$. The effects induced by medial and lateral striatal lesions on intake when the animals were food deprived disappeared when the animals were prefed before trials [lesion $F(3,26)=1.97$, n.s.]

Licking behavior. Although there was a significant effect of lesion group on the overall number of licks per session [see Figure 4B; lesion $F(3,26)=4.10, p<.05$ ], with laterally lesioned animals showing increased licking activity (Dunnett's $t$ test $p<.05$ ), the effect was considerably reduced in prefed animals, compared with fooddeprived animals [food $F(1,26)=11.69, p<.01$ ]. Although prefeeding resulted in a consistent reduction in volume consumption for all lesion groups, licking-activity levels were similar for control and ventral groups during 


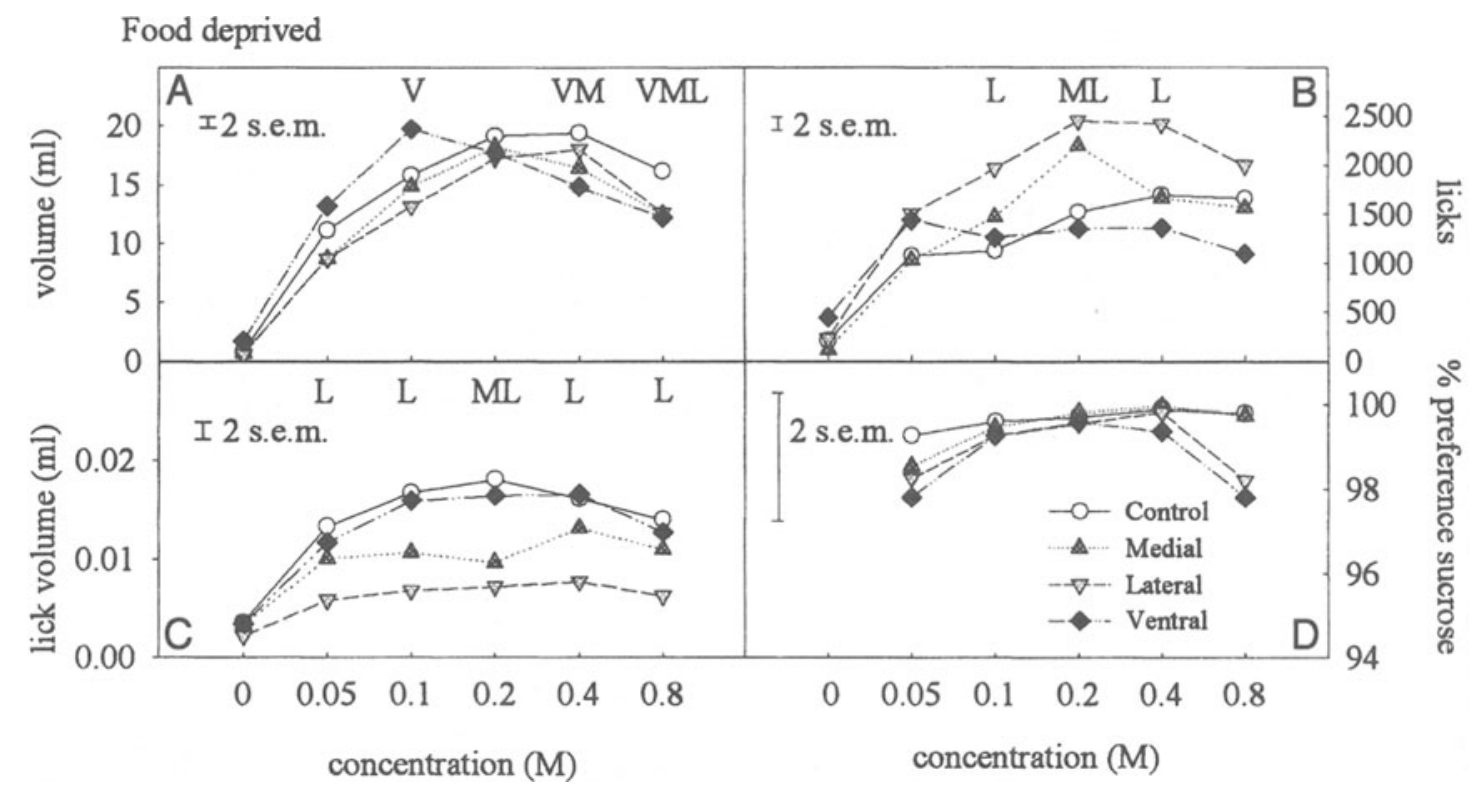

Figure 3. The effects of ventral, medial, and lateral striatal lesions, compared with control, on (A) the volume consumption of sucrose solution, (B) licking activity, (C) lick volume, and (D) percentage preference for sucrose over water. The animals were food deprived before trials. Sucrose was presented in 20 -min, two-tube trials, paired with water. In all cases, the error bars represent $2 S E M$, derived from the relevant interaction term of the analysis of variance. The letters $V$ (ventral), $M$ (medial), and $L$ (lateral) represent significant differences between the relevant lesion group and the control group (Dunnett's $t$ test $p<.05$ ).

the prefeeding and food-deprived trials but were reduced in the lateral and medial lesion groups.

Lick volumes were also significantly affected by prefeeding [see Figure $4 \mathrm{C}$; food $F(1,26)=68.91, p<$ .001 ], and again there were different lesion effects [food $X$ lesion $F(3,26)=11.52, p<.001]$. Control and ventrally lesioned animals showed a marked reduction in lick volume when they were prefed before the trial. Laterally lesioned animals showed almost no difference in lick volume when they were prefed. Nevertheless, there was still a significant difference between lesion groups, and the lick volume of laterally lesioned animals was significantly less than that of control animals [lesion $F(3,26)=3.50$, $p<.05]$. Prefeeding induced a small, but not significant, decrease in the lick volume of medially lesioned animals.

Preference for sucrose over water. Again, there was no effect of any of the striatal lesions on preference for sucrose over water when the animals were prefed [see Figure $4 \mathrm{D}$; lesion $F(3,26)=0.78$, n.s.].

\section{Experiment 3: Affective Contrast Paradigm}

In this experiment, a successive contrast effect was defined as the difference in rats' consumption of a particular concentration of sucrose $(0.05 \mathrm{M})$, dependent upon previous drinking experience during the trial. Figure 5 shows that all groups showed strong positive and negative contrast [contrast $F(2,50)=48.49, p<.001$ ] . Sidák's test was significant for the positive-no-contrast comparison, and the negative-no-contrast comparison, at the $p<.01$ level for both, although the positive contrast effect was much greater.

None of the regional striatal lesions significantly affected the extent of the positive or negative contrast effects [lesion $\times$ contrast $F(6,50)=0.81$, n.s.]. The response of all lesioned animals was similar to that of control animals: increased consumption of sucrose with a positive contrast, and decreased consumption with a negative contrast. For the concentrations used in these trials, one confounding effect could have arisen from slight differences in overall consumption of $0.05 \mathrm{M}$ sucrose by the different lesion groups. However, when the volume of $0.05 \mathrm{M}$ sucrose consumed in 20-min sessions was used as a covariate in the analyses, there were no significant differences in the overall outcome.

\section{DISCUSSION}

\section{Differential Effects of Ventral and Dorsal Striatal Lesions on Sucrose Consumption}

This study has provided evidence that specific fibersparing lesions of regions of the ventral and dorsal striatum produce distinct functional deficits on sucrosedrinking behavior. Most strikingly, lesions of the ventral striatum produced a complete shift in the sucrose concentration-intake curve leftward, in effect causing the rats to react to the sucrose as if it were more concentrated. This 


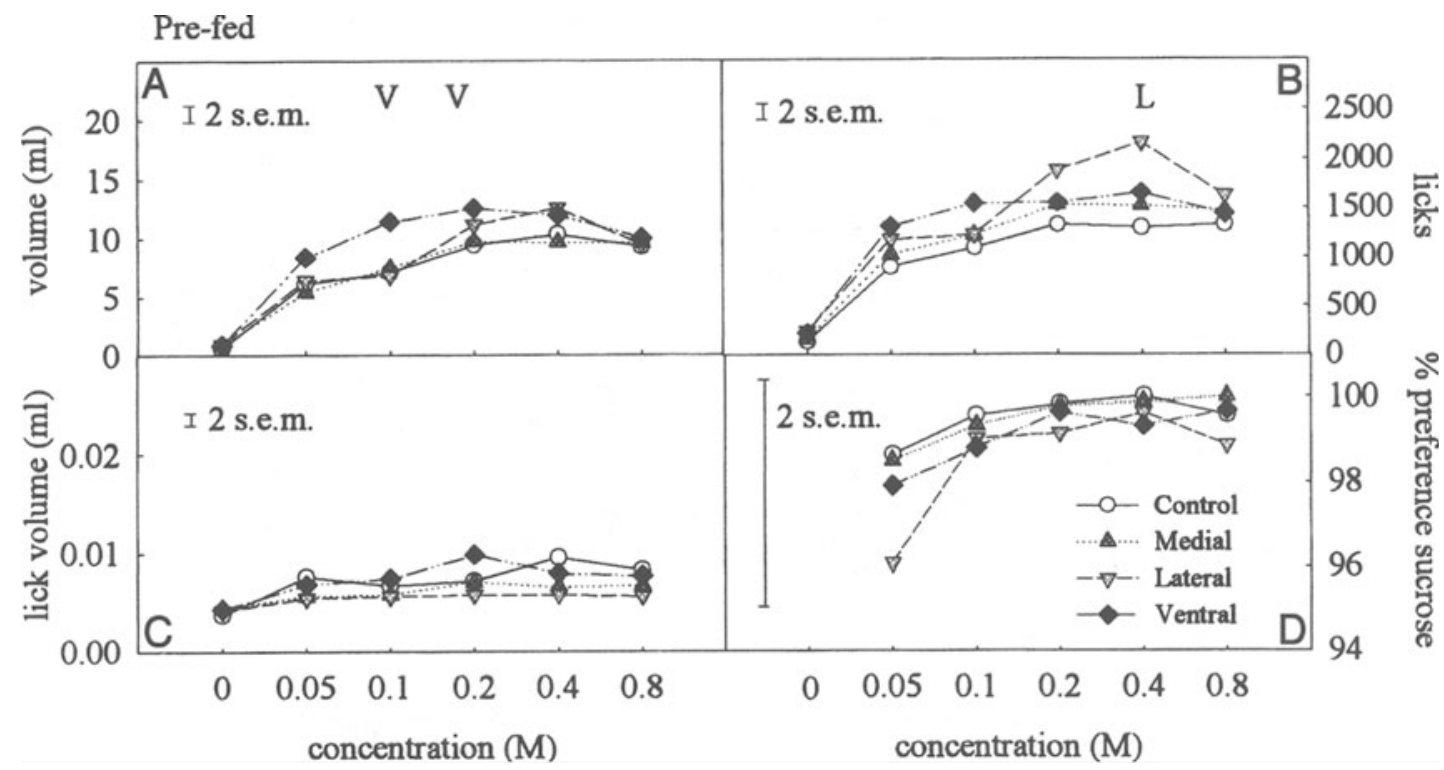

\begin{abstract}
Figure 4. The effects of ventral, medial, and lateral striatal lesions, compared with control, on (A) the volume consumption of sucrose solution, (B) licking activity, (C) lick volume, and (D) percentage preference for sucrose over water. The animals were prefed before trials. Sucrose was presented in $\mathbf{2 0 - m i n , ~ t w o - t u b e ~ t r i a l s , ~ p a i r e d ~ w i t h ~ w a t e r . ~ I n ~ a l l ~ c a s e s , ~}$ the error bars represent $2 S E M$, derived from the relevant interaction term of the analysis of variance. The letters $V$ (ventral) and $L$ (lateral) represent significant differences between the relevant lesion group and the control group (Dunnett's $t$ test $p<.05$ ).
\end{abstract}

shift in intake was relatively independent of hunger level, since it occurred both when the animals were food deprived and when they were prefed before drinking trials. However, ventrally lesioned animals were as sensitive as control animals to changes in food deprivation: Prefeeding reduced their overall consumption, by volume, of sucrose.

In contrast, for both dorso-medial and dorso-lateral striatal lesions, the concentration-intake curve was similar to that of control animals, but intake was significantly reduced. This drinking deficit was also dependent on hunger level, since it was apparent only when the animals were food deprived.

Lateral striatal lesions produced a significant effect on licking behavior, with increased licking activity compared with that of control animals, and an associated reduction in lick volume, when rats were either prefed or food deprived. Volume intake appeared more closely related to licking activity than it did to lick volume. Ventral lesions did not affect either measure of licking significantly, compared with control animals. For both ventral and control groups, licking activity was a relatively stable measure, and lick volume appeared to change with changes in intake. Medial dorsal striatal lesions produced a small effect on licking behavior that was not consistent between concentrations, as well as an effect on lick volume that was intermediate between the effects of lateral and ventral striatal lesions.

There was no effect of ventral, medial, or lateral striatal lesions on positive and negative successive contrast. With the concentrations tested in this experiment, the positive contrast was stronger than the negative contrast for all groups.

\section{Do Ventral Striatal Lesions Enhance "Reward Value"?}

The leftward shift in the concentration-intake function after the rats received excitotoxic ventral striatal lesions is clear evidence of the role of the ventral striatum in certain aspects of consummatory behavior. The shift in intake might be considered an enhancement of "reward value." This contrasts with other studies that have manipulated dopaminergic function within the ventral striatum. For example, Phillips et al. (1991a, 1991b, $1991 \mathrm{c})$ found an effective rightward shift in sucrose consumption, or a reduction of "reward value," following dopamine antagonist infusion into the ventral striatum, which was similar to that found following lesions to nuclei of the amygdala (Kemble \& Schwartzbaum, 1969; Rolls \& Rolls, 1973). However, similar mesolimbic dopamine manipulations in other studies have produced only decreased consumption of weaker solutions, while failing to find increased consumption of more concentrated sucrose (Ikemoto \& Panksepp, 1996; Muscat \& Willner, 1989). Intra-accumbens manipulation of opiate receptors has produced more straightforward effects on sucrose ingestion: Opiate agonists enhance (Zhang \& Kelley, 1997), and opiate antagonists decrease (Kelley et al., 1996), consumption over a whole range of concentrations.

Although the ventral striatum, including the nucleus accumbens, is more conventionally implicated in the appetitive-approach rather than the consummatory phases of rewarded behavior, this study provides very clear evidence that the ventral striatum exerts important controls over the aspects of consummatory behavior related to the 


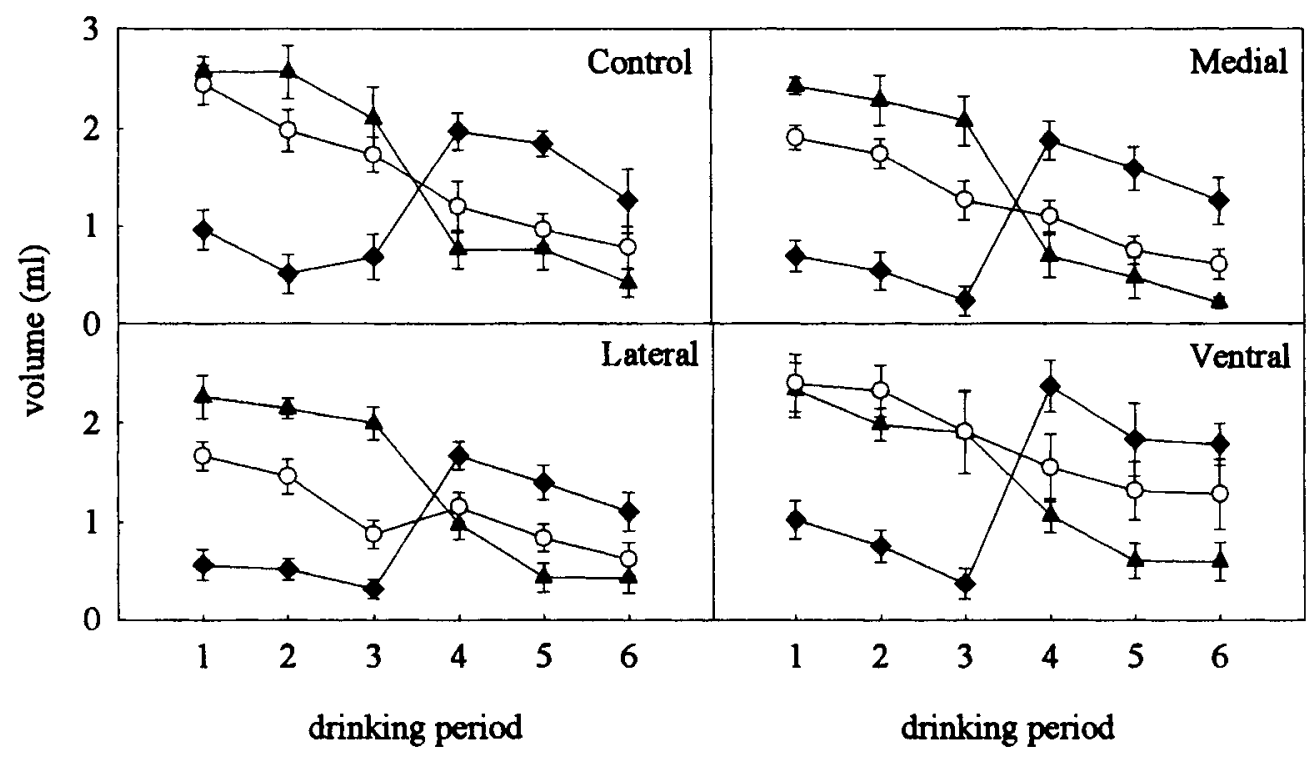

Figure 5. The effects of medial, lateral, and ventral striatal lesions on successive positive (diamond) and negative (triangle) contrast, compared with no contrast sucrose presentations (open circles), during 1-min drinking periods. Sucrose concentration was switched between Drinking Periods 3 and 4. For negative contrast, $0.2 \mathrm{M}$ was switched to $0.05 \mathrm{M}$; for positive contrast, $0.0125 \mathrm{M}$ was switched to $0.05 \mathrm{M}$; and for no contrast, $0.05 \mathrm{M}$ was switched to $0.05 \mathrm{M}$. There were no significant lesion effects on contrast. Error bars represent 2 SEM.

sensory, and possibly, the incentive, qualities of food. Excitotoxic lesions of the ventral striatum produce a shift in response to sucrose rewards that is the opposite of the shift induced by dopamine antagonists and which is independent of internal motivational states such as hunger level.

In this study, there were no other clear incentive motivational deficits induced by ventral striatal lesions. Most importantly, rats with ventral striatal lesions responded to both positive and negative successive contrast in a manner similar to that of control rats. One interpretation of this result would be that relative reward assessment does not appear to be disrupted by lesions to the ventral striatum, including the nucleus accumbens, even though lesions to the corticomedial amygdala eliminated negative consummatory contrast (Becker et al., 1984), and intraamygdala infusions of lidocaine (Salinas et al., 1993) significantly attenuated contrast responses.

However, whereas traditional negative contrast effects are generally thought to reflect affective reactions (see Flaherty, 1996), it is possible that the procedure used in the present study does not do so. The relatively short periods between the last experience with the preshift solution and the first experience with the postshift solution could allow sensory factors to become involved, in a manner analogous to simultaneous contrast (Flaherty, 1996).

There were no obvious motor impairments as a result of ventral striatal lesions, with no differences in the overall level of licking behavior, and there were no differences in lick volume or efficiency between the controls and the ventrally lesioned animals. The ventral striatum appears to be more closely involved in the response to sucrose than in the performance of the consummatory response itself.

Although this dissociation between appetitive and consummatory behaviors is, in general, consistent with previous hypotheses, it is surprising and seemingly paradoxical that ventral striatal lesions enhance the heightened response to sucrose. This finding is clearly in contrast to those of some studies of the effects of dopaminergic manipulations. Our results are paralleled by the findings of similar patterns of the effect of manipulations of the ventral striatum on progressive ratio performance, which is an operant measure of motivation (Bowman \& Brown, 1998; Salamone, Aberman, \& Ward, 1997). The findings may be explained by postulating a disenabling, or gating, role for the ventral striatum on motivated behavior mediated by the structure, which is disinhibited by mesolimbic dopamine activity. These data thus allow a reinterpretation of the role of the nucleus accumbens itself in motivation.

\section{Motor or Motivational \\ Impairments of Dorsal Striatal Lesions?}

This study also provides evidence of the effects of both lateral and medial dorsal striatal lesions on consummatory responses to sucrose. Both lesions resulted in a reduction in the volume of sucrose that was consumed, although this difference was greater for higher concentrations, especially with medial striatal lesions, a finding which could indicate the possibility of a motivational effect (e.g., increased aversion). The effects of dorsal striatal lesions 
were attenuated by prefeeding, probably because lower licking rates masked the deficits. However, neither lateral nor medial striatal lesions had any effect on positive or negative contrast.

Lateral striatal lesions reduced volume intake at all concentrations. This result, coupled with the change in licking behavior, could indicate that there was a motoric deficit, rather than a motivational deficit, associated with the lateral lesions. Specifically, the large increase in licking activity and consequent reduction in lick volume was not found in animals with medial or ventral striatal lesions, where lesioned animals' licking behavior was similar to that of control animals. Neurons connected with oral and facial movement are located in the lateral striatum (Mittler et al., 1994), and there is evidence that manipulations of the lateral striatum-in particular, the ventral lateral striatum (VLS) can produce stereotypically rapid oral responses. Both amphetamine (Kelley et al., 1989) and 5-HT (Yeghiayan, Kelley, Kula, Campbell, \& Baldessarini, 1997) infusions into the VLS induce stereotyped orofacial behaviors such as increased gnawing, and it is possible that manipulations of the VLS could result in enhanced licking behavior when the animal is provided with the opportunity to lick at a sucrose source. Ventral striatal lesions did not produce any significant effects on the rats' overall licking behavior. The effects of schedule-induced polydipsia (SIP) are consistent with our results. In SIP, periodic presentation of small food pellets to food-deprived animals resulted in excess drinking that could not be explained by physiological fluid deficits or superstitious behavior. Mesolimbic dopamine depletion reduced this drinking behavior without affecting normal drinking (Robbins \& Koob, 1980). Mesostriatal dopamine depletion was not as effective in reducing SIP, and, although the volume that was ingested decreased slightly, licking behavior actually increased, a result that indicated a probable effect on motor efficiency (Mittleman, Whishaw, Jones, Koch, \& Robbins, 1990).

The effects of medial striatal lesions were not as clear cut in this study. Volume consumption was reduced, but only following food deprivation. However, medially lesioned animals showed a far greater reduction in consumption at higher concentrations of sucrose; they are on the downward slope of the intake-concentration function. This may indicate an enhanced aversive response to increasing concentrations of sucrose. However, there was no effect of the medial striatal lesions on successive positive or negative contrast. Motoric impairments were not as clear as they were with lateral striatal lesions. Thus, medial lesions may produce a combination of the features of ventral and lateral lesions.

In summary, this study has revealed clear functional differences in sucrose drinking after rats received different lesions of the striatum, a finding that supports the hypothesis of a dorsal striatal involvement in motor capacity and a ventral striatal mediation of aspects of motivational control. However, our analysis supports the need for a reappraisal of the previous role of the ventral striatum in motivational processes.

\section{REFERENCES}

Alexander, G. E., \& Crutcher, M. D. (1990). Functional architecture of basal ganglia circuits: Neural substrates of parallel processing. Trends in Neurosciences, 13, 266-271.

AlHEID, G. F., \& Heimer, L. (1998). New perspectives in basal forebrain organization of special relevance for neuropsychiatric disorders: The striatopallidal, amygdaloid, and corticopetal components of substantia innominata. Neuroscience, 27, 1-39.

BaKshi, V. P., \& Kelley, A. E. (1991a). Dopaminergic regulation of feeding behavior: I. Differential effects of haloperidol microinfusion into three striatal subregions. Psychobiology, 19, 223-232.

BAKSHI, V. P., \& KELLEY, A. E. (1991b). Dopaminergic regulation of feeding behavior: II. Differential effects of amphetamine microinfusion into three striatal subregions. Psychobiology, 19, 233-242.

Balleine, B., \& Killcross, S. (1994). Effects of ibotenic acid lesions of the nucleus accumbens on instrumental action. Behavioural Brain Research, 65, 181-193.

Becker, H. C., Jarvis, M. F., Wagner, G. C., \& Flaherty, C. F. (1984). Medial and lateral amygdalectomy differentially influences consummatory negative contrast. Physiology \& Behavior, 33, 707712 .

Berridge, K. C., \& Cromwell, H. C. (1990). Motivationalsensorimotor interaction controls aphagia and exaggerated treading after striatopallidal lesions. Behavioral Neuroscience, 104, 778-795.

Berridge, K. C., \& Robinson, T. E. (1998). What is the role of dopamine in reward: Hedonic impact, reward learning or incentive salience? Brain Research Reviews, 28, 309-369.

Bowman, E. M., \& Brown, V. J. (1998). Effects of excitotoxic lesions of the rat ventral striatum on the perception of reward cost. Experimental Brain Research, 123, 439-448.

Brown, V. J., \& RobBins, T. W. (1989). Elementary processes of response selection mediated by distinct regions of the striatum. Journal of Neuroscience, 9, 3760-3765.

Burns, L. H., Annett, L., Kelley, A. E., Everitt, B. J., \& Robbins, T. W. (1996). Effects of lesions to amygdala, ventral subiculum, medial prefrontal cortex, and nucleus accumbens on the reaction to novelty: Implication for limbic-striatal interactions. Behavioral Neuroscience, 110, 60-73.

Cousins, M. S., \& Salamone, J. D. (1994). Nucleus accumbens dopamine depletions in rats affect relative response allocation in a novel cost/benefit procedure. Pharmacology, Biochemistry \& Behavior, 49, 85-91.

Cousins, M. S., Sokolowski, J. D., \& Salamone, J. D. (1993). Different effects of nucleus accumbens and ventrolateral striatal dopamine depletions on instrumental response selection in the rat. Pharmacology, Biochemistry \& Behavior, 46, 943-951.

DUNNETT, S. B., \& IVERSEN, S. D. (1980). Regulatory impairments following selective kainic acid lesions of the neostriatum. Behavioural Brain Research, 1, 497-506.

DunNeTt, S. B., \& RobBins, T. W. (1992). The functional role of mesotelencephalic dopamine systems. Biological Reviews of the Cambridge Philosophical Society, 67, 491-518.

EveritT, B. J. (1990). Sexual motivation: A neural and behavioral analysis of the mechanisms underlying appetitive copulatory responses of male rats. Neuroscience \& Biobehavioral Reviews, 14, 217-232.

FlaherTy, C. F. (1982). Incentive contrast: A review of behavioral changes following shifts in reward. Animal Learning \& Behavior, 10, 409-440.

Flaherty, C. F. (1996). Incentive relativity (Problems in the Behavioural Sciences, Vol. 15). Cambridge: Cambridge University Press.

Flaherty, C. F., Grigson, P. S., Demetrikopoulos, M. K., Weaver, M. S., KRauss, K. L., \& Rowan, G. A. (1990). Effect of serotonergic drugs on negative contrast in consummatory behavior. Pharmacology, Biochemistry \& Behavior, 36, 799-806. 
Flaherty, C. F., Rowan, G. A., Emerich, D. F., \& Walsh, T. J. (1989). Effects of intrahippocampal administration of colchicine on incentive contrast and on radial maze performance. Behavioral Neuroscience, 103, 319-328.

Grigson, P. S., Spector, A. C., \& Norgren, R. (1994). Lesions of the pontine parabrachial nuclei eliminate successive negative contrast effects in rats. Behavioral Neuroscience, 108, 714-723.

IKemoto, S., \& PANKSEPP, J. (1996). Dissociations between appetitive and consummatory responses by pharmacological manipulations of reward-relevant brain regions. Behavioral Neuroscience, 110, 331-345.

KeLley, A. E., Bless, E. P., \& SWANSON, C. J. (1996). Investigation of the effects of opiate antagonists infused into the nucleus accumbens on feeding and sucrose drinking in rats. Journal of Pharmacology \& Experimental Therapeutics, 278, 1499-1507.

Kelley, A. E., Gauthier, A. M., \& Lang, C. G. (1989). Amphetamine microinjections into distinct striatal subregions cause dissociable effects on motor and ingestive behavior. Behavioural Brain Research, 35, 27-39.

Kemble, E. D., \& Schwartzbaum, J. S. (1969). Reactivity to taste properties of solutions following amygdaloid lesions. Physiology \& Behavior, 4, 981-985.

KоOB, G. F. (1992). Dopamine, addiction and reward. Seminars in the Neurosciences, 4, 139-148.

KOOB, G. F., \& NESTLER, E. J. (1997). The neurobiology of drug addiction. Journal of Neuropsychiatry \& Clinical Neuroscience, 9, 482 497.

Koob, G. F., Riley, S. J., SMith, S. C., \& Robbins, T. W. (1978). Effects of 6-hydroxydopamine lesions of the nucleus accumbens septi and olfactory tubercle on feeding, locomotor activity and amphetamine anorexia in rats. Journal of Comparative \& Physiological Psychology, 92, 917-927.

Marshall, J. F., Richardson, J. S., \& Teitelbaum, P. (1974). Nigrostriatal bundle damage and the lateral hypothalamic syndrome. Journal of Comparative \& Physiological Psychology, 87, 808-830.

MitCHELL, J. B. (1994). Involvement of mesolimbic dopamine neurons in sexual behaviors: Implications for the neurobiology of motivation. Reviews in the Neurosciences, 55, 615-620.

Mittleman, G., WhishaW, I. Q., Jones, G. H., KoCh, M., \& Robbins, T. W. (1990). Cortical, hippocampal and striatal mediation of scheduleinduced behaviors. Behavioural Neuroscience, 104, 399-409.

Mittler, T., Cho, J., Peoples, L. L., \& West, M. O. (1994). Representation of the body in the lateral striatum of the freely moving rat: Single neurons related to licking. Experimental Brain Research, 98, 163-167.

MuSCAT, R., \& WillnER, P. (1989). Effects of dopamine antagonists on sucrose consumption and preference. Psychopharmacology, 99, 98102.

Phillips, G., Willner, P., \& Muscat, R. (1991a). Anatomical substrates for neuroleptic-induced reward attenuation and neurolepticinduced response decrement. Behavioural Pharmacology, 2, 129-141.

Phillips, G., Willner, P., \& Muscat, R. (1991b). Reward-dependent suppression or facilitation of consummatory behaviour by raclopride. Psychopharmacology, 105, 355-360.
Phillips, G., Willner, P., \& Muscat, R. (1991c). Suppression or facilitation of operant behaviour by raclopride dependent on concentration of sucrose reward. Psychopharmacology, 105, 239-246.

Robbins, T. W., CADor, M., TAylor, J. R., \& EveritT, B. J. (1989). Limbic-striatal interactions in reward-related processes. Neuroscience \& Biobehavioral Reviews, 13, 155-162.

Robbins, T. W., \& EveritT, B. J. (1992). Functions of dopamine in the dorsal and ventral striatum. Seminars in the Neurosciences, 4, 119. 127.

Robiins, T. W., \& EveritT, B. J. (1996). Neurobehavioural mechanisms of reward and motivation. Current Opinions in Neurobiology, 6, 228-236.

Robbins, T. W., \& Коов, G. F. (1980). Selective disruption of displacement behavior by lesions of the mesolimbic dopamine system. Nature, 285, 409-412.

RoLls, B. J., \& RoLls, E. T. (1973). Effects of lesions in the basolateral amygdala on fluid intake in the rat. Journal of Comparative \& Physiological Psychology, 83, 240-247.

Salamone, J. D., Aberman, J., \& Ward, S. J. (1997). The involvement of accumbens dopamine in behavioral dynamics: Studies of progressive ratio responding for food reinforcement. Society for Neuroscience Abstracts, 23, 1359.

Salamone, J. D., Cousins, M. S., \& SNyder, B. J. (1997). Behavioral functions of nucleus accumbens dopamine: Empirical and conceptual problems with the anhedonia hypothesis. Neuroscience \& Biobehavioral Reviews, 21, 341-359.

Salinas, J. A., Packard, M. G., \& McGaugh, J. L. (1993). Amygdala modulates memory for changes in reward magnitude: Reversible post-training inactivation with lidocaine attenuates the response to a reduction in reward. Behavioural Brain Research, 59, 153-159.

UNGERSTEDT, U. (1971). Adipsia and aphagia after 6-hydroxydopamine-induced degeneration of the nigro-striatal dopamine system. Acta Physiologica Scandinavica, 367, 95-122.

Whishaw, I. Q., Mittleman, G., BunCh, S. T., \& Dunnett, S. B. (1987). Impairments in the acquisition, retention and selection of spatial navigation strategies after medial caudate-putamen lesions in rats. Behavioural Brain Research, 24, 125-138.

WiSE, R. A., \& BozARTH, M. A. (1987). A psychomotor stimulant theory of addiction. Psychological Review, 94, 469-492.

Yeghiayan, S. K., Kelley, A. E., Kula, N. S., Campbell, B. A., \& BALDESSARINI, R. J. (1997). Role of dopamine in behavioral effects of serotonin microinjected into rat striatum. Pharmacology, Biochemistry \& Behavior, 56, 251-259.

ZhANG, M., \& KELLEY, A. E. (1997). Opiate agonists microinjected into the nucleus accumbens enhance sucrose drinking in rats. Psychopharmacology, 132, 350-360.

Zigmond, M. J., \& STRICKER, E. M. (1972). Deficits in feeding behavior after intraventricular injection of 6-hydroxydopamine in rats. Science, 177, 1211-1214.

(Manuscript received October 13, 1998 revision accepted January 25,1999 .) 